\title{
Reconstruction of signal phases for signals closer than the DFT frequency resolution
}

\author{
Christian Schiffer and Andreas R. Diewald
}

Laboratory of Radar Technology and Optical Systems, Trier University of applied sciences, 54296 Trier, Germany

Correspondence: Christian Schiffer (c.schiffer@hochschule-trier.de)

Received: 13 February 2021 - Revised: 10 June 2021 - Accepted: 15 June 2021 - Published: 17 December 2021

\begin{abstract}
Radar signal processing is a promising tool for vital sign monitoring. For contactless observation of breathing and heart rate a precise measurement of the distance between radar antenna and the patient's skin is required. This results in the need to detect small movements in the range of $0.5 \mathrm{~mm}$ and below. Such small changes in distance are hard to be measured with a limited radar bandwidth when relying on the frequency based range detection alone. In order to enhance the relative distance resolution a precise measurement of the observed signal's phase is required. Due to radar reflections from surfaces in close proximity to the main area of interest the desired signal of the radar reflection can get superposed. For superposing signals with little separation in frequency domain the main lobes of their discrete Fourier transform (DFT) merge into a single lobe, so that their peaks cannot be differentiated. This paper evaluates a method for reconstructing the phase and amplitude of such superimposed signals.
\end{abstract}

\section{Introduction}

Radar appliances are a promising platform for contactless vital sign monitoring. Due to regulations the usable bandwidth and hence the distance resolution is limited. With signal analysis based on the Fourier transform this results in limitations of the maximum achievable frequency and distance resolution. Algorithms like estimation of signal parameters via rotational invariance techniques (ESPRIT) or MUltiple SIgnal Classification (MUSIC) allow detection of signal frequencies with much closer limits. They provide frequency estimates or a pseudospectrum, that can be used for peak detection. While those methods provide good signal separation in frequency domain, they do not provide values for signal phase or ampli-

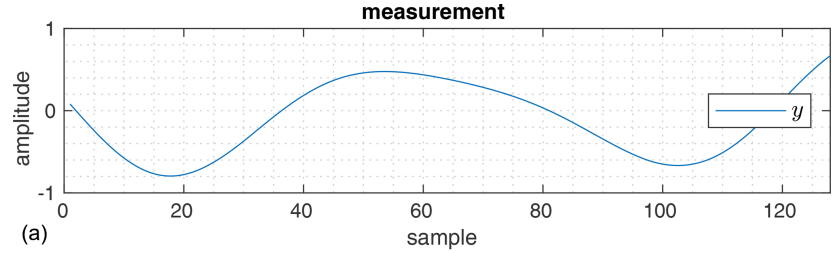

(a)
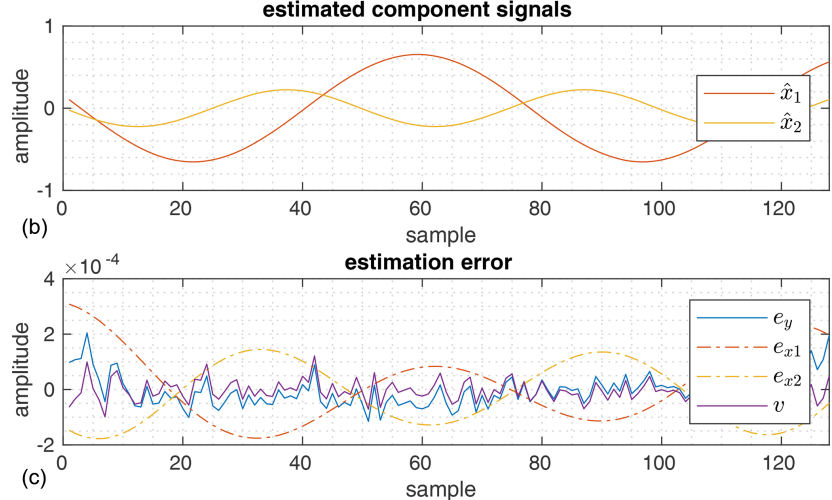

Figure 1. Superposition of signals with similar signal frequencies and their reconstruction in time domain: (a) shows the signal measurement $y$ is shown. Based on this measurement the component signals $\hat{x}_{1}$ and $\hat{x}_{2}$ shown in (b) are estimated. With this the measurement error $e_{y}=y-\hat{x}_{1}-\hat{x}_{2}$ is determined in (c). The actual signal noise $v$, that was used for generating the measurement $y$, is also shown for comparison.

tude. Figure 1 shows the observation of a pair of oscillations, that have similar frequencies.

The corresponding power spectral densities (PSD) displayed in Fig. 2 make the difficulty in determining the correct signal frequencies obvious.

Section 2 presents a way to retrieve the signals' amplitude and phase based on a harmonic signal model, assuming the 


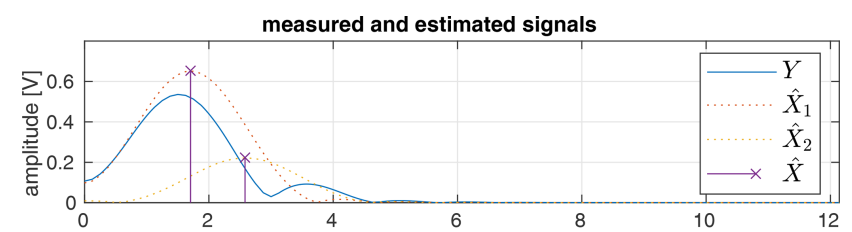

(a)

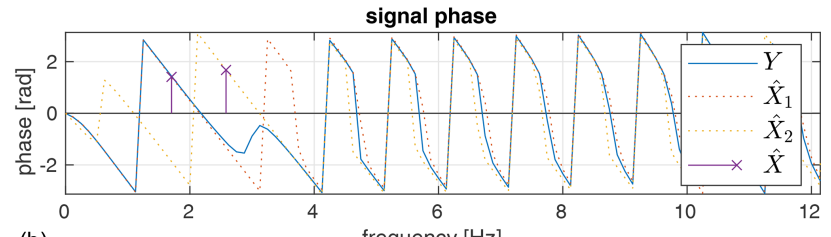

(b)

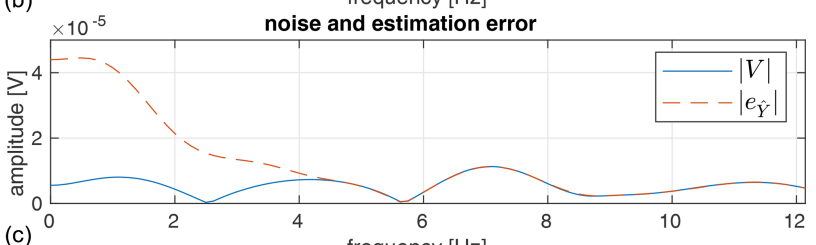

(c)

frequency $[\mathrm{Hz}]$

Figure 2. Superposition of two signals $x_{1}$ and $x_{2}$, that are similar in frequency: (a) shows the power spectral density (PSD) of the measured signal $Y$ and the signal signal noise $V$. The dotted lines show the PSD in (a) and phase of the matching estimated signals $\hat{X}_{1}$ and $\hat{X}_{2}$ in (b). The crosses $\hat{X}$ show amplitude and phase estimates based on the frequency values delivered by an ESPRIT total least squares algorithm. The dashed line in (c) shows the residual error $e_{y}$, when the signal estimates $\hat{X}_{1}$ and $\hat{X}_{2}$ are subtracted from the observed signal.

signals' frequencies can be estimated. The presented method is evaluated in Sect. 3 before a conclusion is discussed in Sect. 4.

\section{Signal parameter estimation}

For a successful phase and amplitude reconstruction an accurate signal model is required. Using a matrix representation of superimposed harmonic signals as described in Sect. 2.1 forms the basis for frequency, phase and amplitude estimation. For the analysis presented in this paper the signal frequencies are determined using estimation of signal parameters via rotational invariance techniques (ESPRIT). However any algorithm could be used, that allows a reasonably precise estimation of signal frequencies. Therefore only the general principle of ESPRIT is described in Sect. 2.2. The phase and amplitude estimation is done using the method described in Sect. 2.3.

\subsection{Harmonic signal model}

A sampled harmonic oscillation $x_{p}$ is completely described by its amplitude $a_{p}$, frequency $f_{p}$ and its initial phase value $\Phi_{p}$ as a function of time $t$ :

$x_{p}(t)=a_{p} \cos \left(2 \pi f_{p} \cdot t+\Phi_{p}\right)$
In discrete signal processing any signal is sampled using a finite sampling frequency $F_{\mathrm{s}}=\frac{1}{T}$. It can be described in multiple equivalent equations:

$$
\begin{aligned}
x_{p}(n)= & \frac{1}{2}\left[\underline{x}_{p}+\underline{x}_{p}^{*}\right] \\
= & \frac{1}{2}\left[a_{p} e^{j 2 \pi f_{p} \cdot T \cdot n+\Phi_{p}}+a_{p} e^{-j 2 \pi f_{p} \cdot T \cdot n-\Phi_{p}}\right] \\
= & a_{p} \cos \left(2 \pi f_{p} \cdot T \cdot n+\Phi_{p}\right) \\
= & a_{p}\left[\cos \left(2 \pi f_{p} T n\right) \cos \left(\Phi_{p}\right)\right. \\
& \left.-\sin \left(2 \pi f_{p} T n\right) \sin \left(\Phi_{p}\right)\right]
\end{aligned}
$$

The superposition of $P$ complex signals $\underline{x}_{p}(n)$

$$
\underline{x}(n)=\sum_{p=1}^{P} \underline{x}_{p}(n)
$$

can be described as follows:

$$
\begin{aligned}
& \underline{a}_{p}=a_{p} \cdot e^{j \Phi_{p}} \\
& \underline{b}_{p}(n)=e^{j 2 \pi f_{p} \cdot T \cdot n} \\
& \Rightarrow \underline{x}(n)=\sum_{p=1}^{P} \underline{a}_{p} \cdot \underline{b}_{p}(n)
\end{aligned}
$$

For real valued signals this expression expands to:

$$
\begin{aligned}
x(n)= & \sum_{p=1}^{P} \frac{1}{2}\left[\underline{a}_{p} \cdot \underline{b}_{p}(n)+\underline{a}_{p}^{*} \cdot \underline{b}_{p}^{*}(n)\right] \\
= & \sum_{p=1}^{P} a_{p}\left[\cos \left(\Phi_{p}\right) \cdot \cos \left(2 \pi f_{p} T n\right)\right. \\
& \left.-\sin \left(\Phi_{p}\right) \cdot \sin \left(2 \pi f_{p} T n\right)\right]
\end{aligned}
$$

\subsection{Frequency estimation}

For the analysis in this paper frequency estimation is done using ESPRIT. Since the method of frequency estimation is exchangeable and not the main scope of this paper, only a rough outline of the ESPRIT algorithm is given in this section (Roy and Kailath, 1989; Ingle et al., 2005).

Based on the harmonic signal model the signal vectors $\underline{x}_{p}$ can be defined

$\underline{\boldsymbol{x}}_{p}=\left[\begin{array}{llll}\underline{x}_{p}(0) & \underline{x}_{p}(1) & \ldots & \underline{x}_{p}(N-1)\end{array}\right]^{\mathrm{T}}$

such that a composite signal matrix $\underline{X}$ can be formed:

$\underline{\mathbf{X}}=\left[\begin{array}{llll}\underline{\boldsymbol{x}}_{1} & \underline{\boldsymbol{x}}_{2} & \cdots & \underline{\boldsymbol{x}}_{P}\end{array}\right]$ 
By splitting the signal matrix $\underline{X}$ into submatrices

$$
\begin{aligned}
& \underline{\mathbf{X}}_{1}=\left[\begin{array}{ll}
\mathbf{I}_{N-1} & \mathbf{0}
\end{array}\right] \underline{\mathbf{X}} \\
& =\left[\begin{array}{cccc}
\underline{x}_{1}(0) & \underline{x}_{2}(0) & \ldots & \underline{x}_{p}(0) \\
\underline{x}_{1}(1) & \underline{x}_{2}(1) & \ldots & \underline{x}_{p}(1) \\
\vdots & \vdots & \ddots & \vdots \\
\underline{x}_{1}(N-2) & \underline{x}_{2}(N-2) & \ldots & \underline{x}_{p}(N-2)
\end{array}\right]
\end{aligned}
$$

and

$$
\begin{aligned}
\underline{\mathbf{X}}_{2} & =\left[\begin{array}{cc}
\mathbf{0} & \mathbf{I}_{N-1}
\end{array}\right] \mathbf{X} \\
& =\left[\begin{array}{cccc}
\underline{x}_{1}(1) & \underline{x}_{2}(1) & \ldots & \underline{x}_{p}(1) \\
\underline{x}_{1}(2) & \underline{x}_{2}(2) & \ldots & \underline{x}_{p}(2) \\
\vdots & \vdots & \ddots & \vdots \\
\underline{x}_{1}(N-1) & \underline{x}_{2}(N-1) & \ldots & \underline{x}_{p}(N-1)
\end{array}\right]
\end{aligned}
$$

the signal frequencies $f_{p}$ are embedded into the diagonal matrix $\boldsymbol{\Phi}$

$\boldsymbol{\Phi}=\operatorname{diag}\left[\begin{array}{llll}e^{j 2 \pi f_{1} T} & e^{j 2 \pi f_{2} T} & \ldots & e^{j 2 \pi f_{P} T}\end{array}\right]$

, that relates both signal matrices $\underline{\mathbf{X}}_{1}$ and $\underline{\mathbf{X}}_{2}$ :

$\underline{\mathbf{X}}_{2}=\underline{\mathbf{X}}_{1} \Phi$

Because the actual signals $\underline{x}_{p}$ and the corresponding signal matrix $\underline{\mathbf{X}}$ are unknown in real applications, for the ESPRIT algorithm a measured signal vector $\boldsymbol{y}=$ $\left[\begin{array}{llll}y(0) & y(1) & \ldots & y(N-1)\end{array}\right]$ is rearranged to generate a data matrix

$\mathbf{Y}=\left[\begin{array}{cccc}y(0) & y(1) & \ldots & y(M-1) \\ y(1) & y(2) & \ldots & y(M) \\ \vdots & \vdots & \ddots & \vdots \\ y(N-M) & y(N-M+1) & \ldots & y(N-1)\end{array}\right]$

. This data matrix is used for singular value decomposition

$\mathbf{Y}=\mathbf{L} \boldsymbol{\Sigma} \mathbf{U}^{\mathrm{H}}$

in order to get the signal subspace $\mathbf{U}_{\mathrm{s}}^{\mathrm{H}}$ from $\mathbf{U}^{\mathrm{H}}$. The signal subspace $\mathbf{U}_{\mathrm{s}}$ itself is split into

$\underline{\mathbf{U}}_{1}=\left[\begin{array}{ll}\mathbf{I}_{P-1} & \mathbf{0}\end{array}\right] \mathbf{U}_{\mathrm{s}}$

and

$\underline{\mathbf{U}}_{2}=\left[\begin{array}{ll}\mathbf{0} & \mathbf{I}_{P-1}\end{array}\right] \mathbf{U}_{\mathrm{s}}$,

so that both matrices are related by the rotation matrix $\Psi$ :

$\mathbf{U}_{2}=\mathbf{U}_{1} \Psi$

The eigenvalues of $\boldsymbol{\Psi}$ correspond to the values in the diagonal matrix $\boldsymbol{\Phi}$, so that the component signal frequencies $f_{p}$ can be acquired.

\subsection{Phase and amplitude reconstruction}

The signal model presented in Sect. 2.1 and a reasonably good estimate of the signal frequencies give the prerequisites for phase and amplitude reconstruction. By writing Eq. (9) as a matrix operation relating the signal vector $\underline{\boldsymbol{x}}$

$\underline{\boldsymbol{x}}=\left[\begin{array}{lllll}\underline{x}(0) & \underline{x}(1) & \cdots & \underline{x}(N-2) & \underline{x}(N-1)\end{array}\right]^{\mathrm{T}}$

and complex amplitude vector $\underline{\boldsymbol{a}}$

$\underline{\boldsymbol{a}}=\left[\begin{array}{lllll}\underline{a}_{1} & \underline{a}_{1}^{*} & \cdots & \underline{a}_{P} & \underline{a}_{P}^{*}\end{array}\right]^{\mathrm{T}}$

a simple matrix operation can be used to describe the sum of an arbitrary number of harmonic oscillations:

$\underline{\boldsymbol{x}}=\underline{\mathbf{B}} \cdot \underline{\boldsymbol{a}}$

With the condition of a known sampling time $T$ and known signal frequencies $f_{p}$ the matrix $\underline{\mathbf{B}}$ is defined according to Eq. (8) for an observation window of $N$ samples:

$\underline{\mathbf{B}}=\left[\begin{array}{cccc}\underline{b}_{1}(0) & \underline{b}_{2}(0) & \cdots & \underline{b}_{P}(0) \\ \underline{b}_{1}(1) & \underline{b}_{2}(1) & \cdots & \underline{b}_{P}(1) \\ \vdots & \vdots & \ddots & \vdots \\ \underline{b}_{1}(N-1) & \underline{b}_{2}(N-1) & \cdots & \underline{b}_{P}(N-1)\end{array}\right]$

Similarly Eq. (11) can be written as a real valued matrix operation relating the signal vector $\boldsymbol{x}$ and a real valued amplitude vector $\boldsymbol{a}$ :

$\boldsymbol{x}=\mathbf{B} \cdot \boldsymbol{a}$

In this case the amplitude vector becomes a composite of the in-phase and the quadrature amplitude values $a_{p, \mathrm{I}}$ and $a_{p, \mathrm{Q}}$ :

$\boldsymbol{a}=\left[\begin{array}{lllll}a_{1, \mathrm{I}} & a_{1, \mathrm{Q}} & \ldots & a_{P, \mathrm{I}} & a_{P, \mathrm{Q}}\end{array}\right]^{\mathrm{T}}$

The real valued matrix $\mathbf{B}$ is defined defined as:

$$
\begin{aligned}
& \mathbf{B}=\left[\begin{array}{ccc}
\cos \left(2 \pi f_{1} T \cdot 0\right) & \sin \left(2 \pi f_{1} T \cdot 0\right) & \cdots \\
\cos \left(2 \pi f_{1} T \cdot 1\right) & \sin \left(2 \pi f_{1} T \cdot 1\right) & \cdots \\
\vdots & \vdots & \ddots \\
\cos \left(2 \pi f_{1} T \cdot[N-1]\right) & \sin \left(2 \pi f_{1} T \cdot[N-1]\right) & \cdots
\end{array}\right. \\
& \left.\begin{array}{ccc}
\cdots & \cos \left(2 \pi f_{P} T \cdot 0\right) & \sin \left(2 \pi f_{P} \cdot 0\right) \\
\cdots & \cos \left(2 \pi f_{P} T \cdot 1\right) & \sin \left(2 \pi f_{P} T \cdot 1\right) \\
\ddots & \vdots & \vdots \\
\cdots & \cos \left(2 \pi f_{P} T \cdot[N-1]\right) & \sin \left(2 \pi f_{P} T \cdot[N-1]\right)
\end{array}\right]
\end{aligned}
$$

Both complex and real matrix definitions can be used to describe a real valued signal vector $\boldsymbol{x}$ and provide a relation to the signal's phase $\Phi_{p}$ and its amplitude $a_{p}$ :

$$
\begin{aligned}
& \underline{a}_{p}=a_{p, \mathrm{I}}-j a_{p, \mathrm{Q}} \\
& \Phi_{p}=\operatorname{Arg}\left(\underline{a}_{p}\right)=-\tan \left(\frac{a_{p, \mathrm{I}}}{a_{p, \mathrm{Q}}}\right) \\
& a_{p}=\left|\underline{a}_{p}\right|=\sqrt{a_{p, \mathrm{I}}^{2}+a_{p, \mathrm{Q}}^{2}}
\end{aligned}
$$

By using a pseudo inverse of $\mathbf{B}$ or $\underline{\mathbf{B}}$ Eqs. (27) and (29) can be solved for the complex and real amplitude vectors $\underline{\boldsymbol{a}}$ 
and $\boldsymbol{a}$ :

$\underline{\mathbf{B}}^{+} \boldsymbol{x}=\underline{a}$

$\mathbf{B}^{+} \boldsymbol{x}=\boldsymbol{a}$

As $\underline{\boldsymbol{a}}$ consists of the complex signal amplitudes $\underline{a}_{p}$, the pseudo inverse $\underline{\mathbf{B}}^{+}$essentially is a reduced form of the discrete Fourier transform:

$\underline{\mathbf{B}}^{+}=\left[\underline{\mathbf{B}}^{\mathrm{H}} \underline{\mathbf{B}}\right]^{-1} \underline{\mathbf{B}}^{\mathrm{H}}$

With an observation $y(n)$ containing some noise $v(n)$

$y(n)=x(n)+v(n)$

this approach results in a least squares solution

$\boldsymbol{a}_{\mathrm{LS}}=\mathbf{B}^{+} \boldsymbol{y}$

that minimizes the quadratic error $\mathbf{Q}_{y}$

$$
\begin{aligned}
\mathbf{Q}_{y} & =\boldsymbol{e}_{y} \boldsymbol{e}_{y}^{\mathrm{H}} \\
& =[\boldsymbol{y}-\boldsymbol{x}][\boldsymbol{y}-\boldsymbol{x}]^{\mathrm{H}}
\end{aligned}
$$

while assuming errors in the transition matrix $\mathbf{Q}_{B}$ being $\mathbf{0}$ :

$$
\begin{aligned}
\mathbf{Q}_{B} & =\boldsymbol{e}_{B} \boldsymbol{e}_{B}^{\mathrm{H}} \\
& =[\hat{\mathbf{B}}-\mathbf{B}][\hat{\mathbf{B}}-\mathbf{B}]^{\mathrm{H}}
\end{aligned}
$$

Due to imperfect knowledge of the observed signal frequencies, an increased error in $\underline{\mathbf{B}}$ and $\mathbf{B}$ respectively is to be expected. Therefore a total least square algorithm to minimize both $\mathbf{Q}_{y}$ and $\mathbf{Q}_{B}$ might be desirable in some applications (Ottersten et al., 1991; Markovsky and Van Huffel, 2007).

\section{Testing}

The methods described in Sect. 2 are tested using a series of randomly generated signals consisting of two real valued oscillations superimposed in each realization. Both the true frequency values used for generating the test signals and frequency estimates acquired by the ESPRIT algorithm are used for phase and amplitude estimation as described in Sect. 2.3.

With the parameters listed in Table 1 random signals for testing are created according to the following equations:

$$
\begin{aligned}
& x_{1}(n, \xi)=a_{1}(\xi) \cdot \cos \left(2 \pi \frac{f_{\mathrm{c}}(\xi)-0.5 \Delta f(\xi)}{F_{\mathrm{s}}} \cdot n+\Phi_{1}(\xi)\right) \\
& x_{2}(n, \xi)=a_{2}(\xi) \cdot \cos \left(2 \pi \frac{f_{\mathrm{c}}(\xi)+0.5 \Delta f(\xi)}{F_{\mathrm{s}}} \cdot n+\Phi_{2}(\xi)\right) \\
& y(n, \xi)=x_{2}(n, \xi)+x_{2}(n, \xi)+v(n, \xi)
\end{aligned}
$$

Figures 1 and 2 display one of the signals, that are generated with the described parameters. The time domain depiction shows both the generated and the reconstructed signals using the measured phase and amplitude. The displayed error

$e_{y}(n)=y(n)-\hat{x}(n)$

represents the difference between the measured signal

$y(n)=x_{1}(n)+x_{2}(n)+v(n)$

and the reconstructed signal:

$\hat{x}(n)=\hat{x}_{1}(n)+\hat{x}_{2}(n)$

The errors $e_{x 1}$ and $e_{x 2}$ are generated accordingly. The frequency domain representation displayed in Fig. 2 shows, that the measurement error $e_{y}(n)$ approximates the actual measurement noise $v(n)$ closely. Furthermore the power leakage from the windowed signals across the frequency spectrum is removed almost entirely. Due to the nature of the least squares algorithm used to derive the complex signal amplitudes, the residual PSD will be close to the sampling noise even with bad frequency estimates.

For further understanding of the limitations connected to the provided method of phase determination first some limitations of the ESPRIT algorithm are presented in Sect. 3.1 representative for a method of frequency estimation. In Sect. 3.2 the results are further investigated in regard to the resulting root mean square error (RMSE).

\subsection{Limits of ESPRIT}

When analyzing the resulting measurements, the limits ESPRIT must be examined. For this purpose a range of randomly distributed signals according to Table 1 is generated and analyzed using ESPRIT. Afterwards the measured oscillations corresponding to distinct signal frequencies are considered valid measurements. The ratio of correctly detected oscillations is displayed in Fig. 3.

As shown in Fig. 3 the rate of correctly detected oscillation frequencies is mostly depending on the frequency separation $\Delta f$ and the signal to noise ratio (SNR) of the sampled signal. Center frequency, input phase and power difference between both oscillations play a minor role.

Notable is the increase of erroneous detections close to $0 \mathrm{~Hz}$ and close to the Nyquist frequency $64 \mathrm{~Hz}$. Below the minimum frequency of $1 \mathrm{~Hz}$ and above of $1 \mathrm{~Hz}$ below the Nyquist frequency the rate of correctly identified frequencies drops to less than 0.5 . With a frequency separation above $0.14 \mathrm{~Hz}$ two oscillations can be separated in with a ratio above 0.5 . At $0.32 \mathrm{~Hz}$ frequency separation this ratio becomes greater than 0.9. Applied to a radar system with a bandwidth of $250 \mathrm{MHz}$, as it is common in $24 \mathrm{GHz}$ radar applications, this could result in a $90 \%$ peak separation rate for reflecting surfaces with a distance of approximately $20 \mathrm{~cm}$.

\subsection{Measurement error analysis}

The set of successfully separated signals is evaluated further. The system's accuracy is analyzed regarding the accuracy in 
Table 1. Testing parameters.

\begin{tabular}{|c|c|c|}
\hline name & value & description \\
\hline$F_{\mathrm{s}}$ & $128 \mathrm{~Hz}$ & Sampling frequency \\
\hline$N_{\mathrm{S}}$ & 128 & Number of samples per ramp \\
\hline$N_{\mathrm{R}}$ & $10^{6}$ & Number of realizations \\
\hline$\xi$ & & realization \\
\hline$v(n, \xi)$ & $\mathrm{WGN}\left(0, \sigma_{v}\right)$ & white Gaussian noise with $10 \lg \left(\sigma_{v}^{2}\right)=-87 \mathrm{~dB}(\approx 14.5$ bit dynamic range $)$ \\
\hline$\Delta f(\xi)$ & {$[0 \mathrm{~Hz}, 1 \mathrm{~Hz}]$} & range of random frequency distance (uniform probability distribution) \\
\hline$f_{\mathrm{c}}(\xi)$ & 1 to $63 \mathrm{~Hz}$ & center frequency \\
\hline$\Phi_{1}(\xi), \Phi_{2}(\xi)$ & {$[0,2 \pi]$} & range of random signal phase (uniform probability distribution) \\
\hline$a_{1}(\xi), a_{2}(\xi)$ & {$\left[10^{-6}, 10^{-0.3}\right]$} & range of random signal amplitude (logarithmic probability distribution) \\
\hline
\end{tabular}

Rate of signal separation
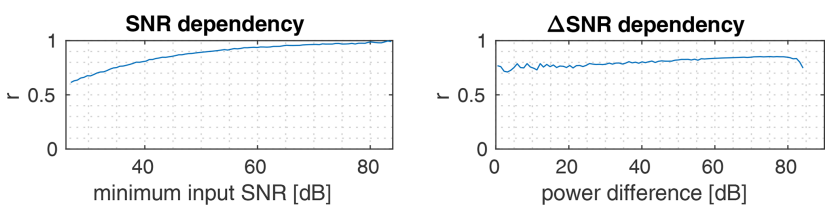

f dependency
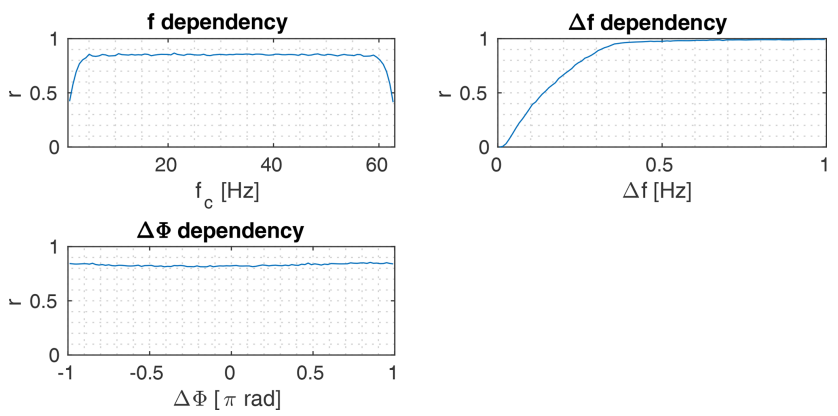

Figure 3. Rate of successful signal separation based on ESPRIT: The number of successfully detected distinct oscillations in relation to the total number of measurements $r=N_{\text {separated }} / N_{\text {total }}$.

Table 2. Summarized results.

\begin{tabular}{ll}
\hline parameter name & value \\
\hline SNR mean & $67.6347 \mathrm{~dB}$ \\
SNR range & 27 to $87 \mathrm{~dB}$ \\
amplitude mean & $-19.4 \mathrm{~dB}$ \\
amplitude range & -60 to $-3 \mathrm{~dB}$ \\
amplitude distribution & $\log$ \\
frequency range & 1 to $63 \mathrm{~Hz}$ \\
$\Delta f$ & $1 \mathrm{~Hz}$ \\
$\sigma_{v}$ & $-87 \mathrm{~dB}$ \\
$\operatorname{RMSE}(\Phi(f))$ & $0.41533 \times 10^{-3} \cdot \pi \mathrm{rad}$ \\
$\operatorname{RMSE}(\Phi(\hat{f}))$ & $20.942 \times 10^{-3} \cdot \pi \mathrm{rad}$ \\
\hline
\end{tabular}

frequency estimation and phase measurement. Table 2 gives a summarized overview of the performed signal simulation.

The distribution of the estimated frequencies root mean square error (RMSE) displayed in Fig. 4 gives further insight into the performance of the utilized ESPRIT algorithm. The observed estimation error of the signal frequency is consis-
$\operatorname{RMSE}(\hat{f})$
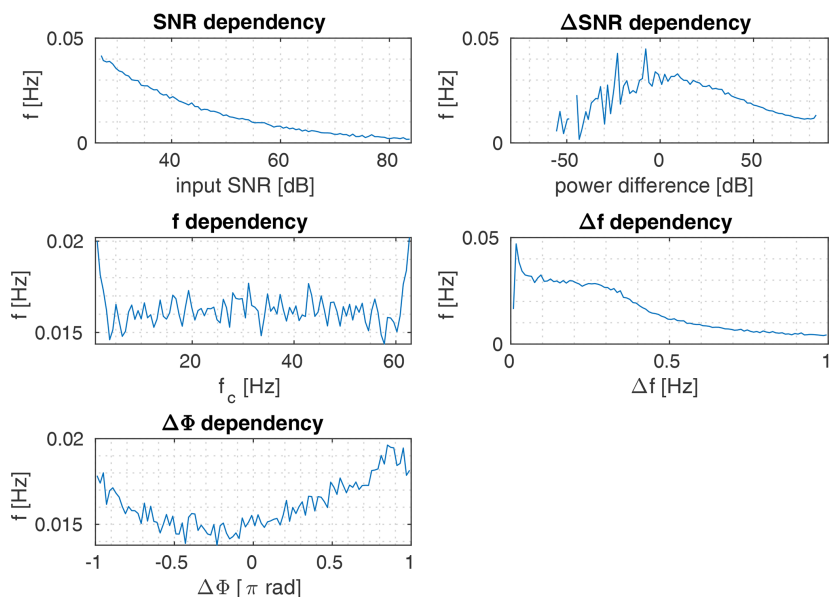

Figure 4. RMSE of the signal frequencies estimated by the ESPRIT method for all successfully separated signals.

tent with the results of successful frequency separation discussed in Sect. 3.1.

The resulting RMSE of the measured signal phase based on the actual signal frequencies is displayed in Fig. 5. The phase error distribution is comparable to the frequency error distribution, though the displayed results must be taken with a grain of salt, because measurements without successful frequency separation are excluded from the statistical RMSE analysis. This especially becomes clear when looking at the $f$ and $\Delta f$ subplots: In the upper and lower ranges of the center frequency $f_{\mathrm{c}}$ and below a frequency separation of $\Delta f=0.4 \mathrm{~Hz}$ the phase error decreases. This is due to measurements with bad signal separation being disregarded. In general the phase estimation error is dependent primarily on the signal's SNR and decreases with increasing SNR and frequency separation.

In comparison to the phase measurement based on the true frequency value, the phase error is two orders of magnitude worse, when it's based on imperfect frequency measurements. Across the complete set of measurements the root mean square error is $0.021 \pi \mathrm{rad}=3.78^{\circ}$. Transferred to a 


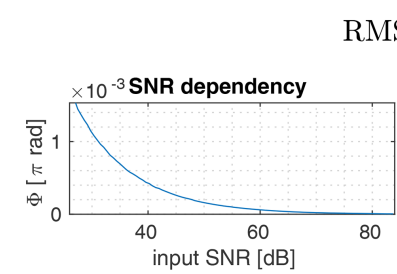

$\operatorname{RMSE}(\Phi(f))$
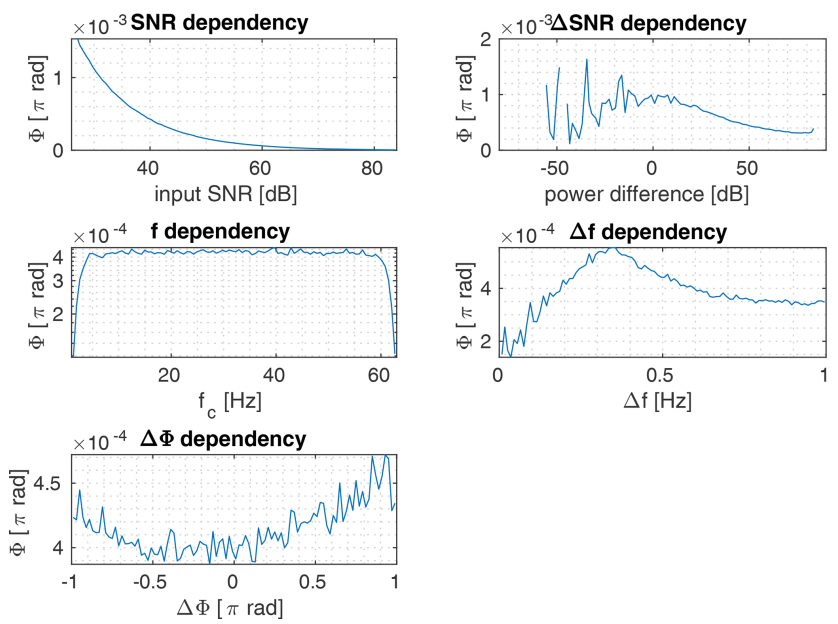

Figure 5. RMSE of the signal phase derived from the measured signal using the true signal frequency for the successfully separated signals.

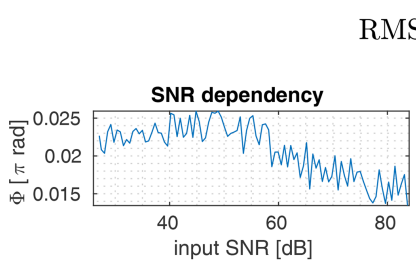

$\operatorname{RMSE}(\Phi(\hat{f}))$
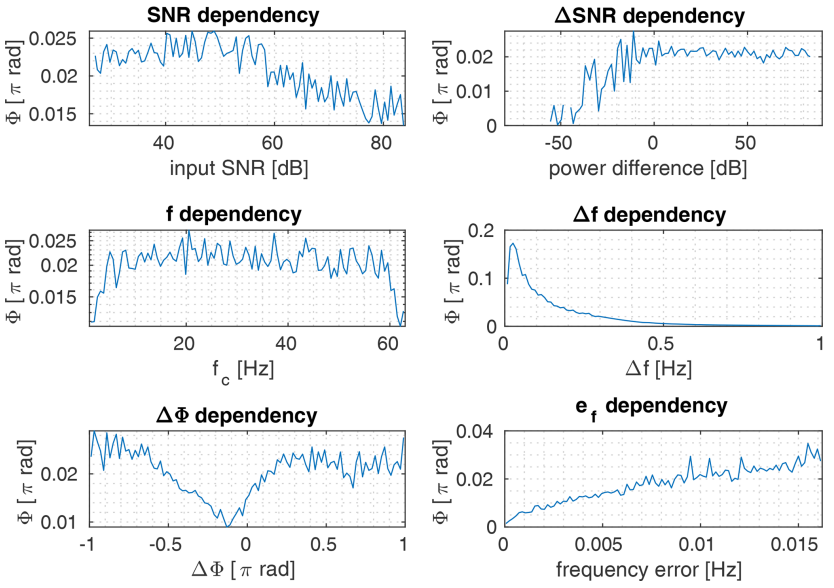

Figure 6. RMSE of the signal phase derived from the measured signal using the signal frequency estimated by the ESPRIT method for the successfully separated signals.

$24 \mathrm{GHz}$ radar application with a wavelength of $12 \mathrm{~mm}$ this transfers to a relative distance error of $63 \mu \mathrm{m}$. While the dependency on the signal to noise ratio (SNR) is almost negligible, the dependency on the frequency separation $\Delta f$ becomes more obvious. As expected, an error in the estimated frequency results in an increased phase error.

\section{Conclusions}

In conclusion the method described in Sect. 2.3 represents a useful addition to frequency estimations like MUSIC or ESPRIT. While depending on the quality of the underlying frequency estimation, a very promising level of accuracy in phase measurement can be reached. Given that the analysis in this paper are done purely on synthetic data, further investigations using real world data are required.

Code availability. Code will be uploaded to: https://gitlab.com/ iffer/closesignalseparation (Schiffer, 2021).

Data availability. All data used is generated using the matlab random number generator.

Author contributions. ARD conceived the parent idea of decomposing overlapping signals, that are close in frequency domain. CS implemented, tested and refined the reconstruction algorithm.

Competing interests. The authors declare that they have no conflict of interest.

Disclaimer. Publisher's note: Copernicus Publications remains neutral with regard to jurisdictional claims in published maps and institutional affiliations.

Special issue statement. This article is part of the special issue "Kleinheubacher Berichte 2020".

Review statement. This paper was edited by Madhu Chandra and reviewed by two anonymous referees.

\section{References}

Ingle, V., Kogon, S., and Manolakis, D.: Statisical and Adaptive Signal Processing, Artech House, Norwood, USA, 816 pp., 2005.

Markovsky, I. and Van Huffel, S.: Overview of total leastsquares methods, Signal Processing, 87, 2283-2302, https://doi.org/10.1016/j.sigpro.2007.04.004, 2007.

Ottersten, B., Viberg, M., and Kailath, T.: Performance analysis of the total least squares ESPRIT algorithm, IEEE T. Signal Process., 39, 1122-1135, https://doi.org/10.1109/78.80967, 1991.

Roy, R. and Kailath, T.: ESPRIT-estimation of signal parameters via rotational invariance techniques, IEEE T. Acoust. Speech, 37, 984-995, https://doi.org/10.1109/29.32276, 1989.

Schiffer, C.: Code to "Reconstruction of signal phases for signals closer than the DFT frequency resolution", GitLab [code], available at: https://gitlab.com/iffer/closesignalseparation, last access: 23 September 2021. 